\title{
Massively parallel pooled screening reveals genomic determinants of nanoparticle-cell interactions
}

\author{
Natalie Boehnke ${ }^{1,2} \uparrow$, Joelle P. Straehla ${ }^{1,2,3,4} \uparrow$, Hannah C. Safford ${ }^{1}$, Mustafa Kocak ${ }^{2}$, Matthew G. \\ Rees $^{2}$, Melissa Ronan ${ }^{2}$, Danny Rosenberg ${ }^{2}$, Charles H. Adelmann ${ }^{5,6,7}$, Raghu R. Chivukula ${ }^{6,8}$, \\ Jaime H. Cheah ${ }^{1}$, Hojun Li ${ }^{1,3,4}$, Jennifer A. Roth ${ }^{2}$, Angela N. Koehler ${ }^{1,2,9}$, Paula T. \\ Hammond $^{1,10 *}$
}

\section{Affiliations:}

${ }^{1}$ Koch Institute for Integrative Cancer Research, Massachusetts Institute of Technology; Cambridge, MA, USA.

${ }^{2}$ Broad Institute of MIT and Harvard; Cambridge, MA, USA.

${ }^{3}$ Department of Pediatric Oncology, Dana-Farber Cancer Institute; Boston, MA, USA.4

${ }^{4}$ Division of Pediatric Hematology/Oncology, Boston Children's Hospital; Boston, MA, USA.

${ }^{5}$ Cutaneous Biology Research Center, Massachusetts General Hospital Department of Dermatology, Harvard Medical School; Boston, MA, USA.

${ }^{6}$ Whitehead Institute for Biomedical Research; Cambridge, MA, USA

${ }^{7}$ Department of Biology, Massachusetts Institute of Technology; Cambridge, MA, USA.

${ }^{8}$ Division of Pulmonary and Critical Care Medicine, Department of Medicine, Massachusetts General Hospital; Boston, MA, USA.

${ }^{9}$ Department of Biological Engineering, Massachusetts Institute of Technology; Cambridge, MA, USA.

${ }^{10}$ Department of Chemical Engineering, Massachusetts Institute of Technology; Cambridge, MA, USA.

*Corresponding author. Email: hammond@mit.edu

$\dagger$ These authors contributed equally to this work 


\begin{abstract}
:
To accelerate the translation of cancer nanomedicine, we hypothesize that integrated genomic screens will improve understanding of the cellular processes governing nanoparticle trafficking. We developed a massively parallel high-throughput screening method leveraging barcoded, pooled cancer cell lines annotated with multi-omic data to investigate cell association patterns across a nanoparticle library spanning a range of formulations with clinical potential. This approach identified both the materials properties and cell-intrinsic features mediating nanoparticlecell association. Coupling the data with machine learning algorithms, we constructed genomic nanoparticle trafficking networks and identified nanoparticle-specific biomarkers. We engineered cell lines to validate SLC46A3 as a biomarker whose expression inversely predicts liposomal nanoparticle uptake both in vitro and in vivo. Our work establishes the power of massively parallel pooled cell screens for nanoparticle delivery and enables the identification and utilization of biomarkers to rationally design nanoformulations for specific patient populations.
\end{abstract}

\title{
Main Text:
}

Nanoparticle (NP)-based therapeutics have enormous potential for personalized cancer therapy as they can encapsulate a range of therapeutic cargos including small molecules, biologics and, more recently, nucleic acids. $(1,2)$ Therapy-loaded NPs can be designed to prevent undesired degradation of the cargo, increase circulation time, and direct drugs specifically to target tumors. There have been notable successes in clinical translation of nanomedicines, including liposomal formulations of doxorubicin (Doxil) and irinotecan (Onivyde ${ }^{\circledR}$ ).(3) These formulations extend the half-life of the active agent and have the potential to lower toxicity, but do not efficiently accumulate in tumors. $(4,5)$

Efforts to improve NP accumulation in tumors via active targeting motifs have been met with limited success, both in the laboratory and the clinic. $(1,6)$ While progress has been made in understanding how specific physical and chemical NP properties affect trafficking and uptake, comprehensive evaluation of multiple NP parameters in combination has thus far been elusive. Additionally, tissue and cellular heterogeneity make it prohibitively challenging to gain a holistic understanding of which NP properties dictate successful trafficking and drug delivery. $(7,8)$ Once these NP parameters are considered in combination, the number of unique 
formulations to test increases exponentially, particularly as comparisons across several systems need to be drawn. A further barrier is the need to adapt the nanoparticle formulation of each encapsulated therapy for a given drug or target, as each formulation has its own unique biological fate.(8) As therapies continue to increase in molecular complexity, new nanocarrier formulations capable of delivering these entities will need to be developed and examined for their unique trafficking properties.

We and others have designed panels of NPs to elucidate the structure-function relationships to cellular targeting and uptake.(9-12) However, there is a need to equally consider the influence of biological heterogeneity on these interactions. In the era of precision medicine, with the desire to deliver molecularly targeted and gene-based therapies to specific subcellular compartments within cancer cells, it is imperative to holistically probe the structure-function relationship of NPs as they relate to cellular interactions.

Inspired by recent advancements in cancer genomics,(13) we postulated that applying these same techniques to the study of cancer nanomedicine would uncover both the cell- and NPspecific features mediating efficient targeting and delivery. The combination of pooled screening with multi-omic annotation has accelerated target discovery and uncovered previously unrecognized mechanisms of action in small molecule screens. Specifically, in the Profiling Relative Inhibition in Mixtures (PRISM) method, DNA-barcoded mixtures of cells have recently been used for multiplexed viability screening. In cell line pools grouped by doubling time, 500 barcoded cell lines have been screened against tens of thousands of compounds to identify genotype-specific cancer vulnerabilities. $(14,15)$

To comprehensively capture pan-cancer complexities and enable the statistical power to link NP association with cell intrinsic characteristics, we developed a competitive phenotypic screen to assess associations of a curated NP library across hundreds of cancer cell lines simultaneously. By pooling and plating 488 DNA barcoded cancer cell lines in a single well, we screened the interactions of a range of NP formulations with varied core compositions, surface chemistries, and diameters. We observed that NP core composition has a dominating influence on cell-specific interactions of the studied parameters. Coupling our biomarker findings with k-means clustering, we constructed genomic interaction networks associated with NP engagement, enabling the identification and connection of genes associated with the 
binding, recognition, and subcellular trafficking of distinct NP formulations. Moreover, through the use of univariate analyses and random forest algorithms, we identified that the gene SLC46A3 holds significant value as a predictive, NP-specific biomarker. We further validated SLC46A3 as a negative regulator of liposomal NP uptake in vitro and in vivo. The strategy outlined herein identifies cellular features underlying nanoparticle engagement, adding a new dimension to the study of cancer nanomedicine.

\section{nanoPRISM: screening nanoparticle association with pooled cell lines}

To screen hundreds of cancer cell lines simultaneously for NP-cancer cell line association patterns, we cultured pooled PRISM cells and incubated them with fluorescent NPs. We then implemented a fluorescence-activated cell sorting (FACS) adaptive gating strategy to sort cell populations into four bins (quartiles, A-D) based on fluorescence signal as a proxy for the extent of NP-cell association (Figure 1A). Experimental parameters were optimized to ensure sufficient cell number and barcode representation post-cell sorting (Figure S1) and NPs were incubated for 4 and 24 hours.

For this screen, we designed a modular NP library to capture the effects of NP core composition, surface chemistry, and size on cell interactions. This panel of 35 NPs encompassed both clinical and experimental formulations. Specifically, anionic liposomes were formulated and electrostatically coated with cationic poly-L-arginine (PLR) followed by a series of polyanions.(16-20) The polyanions were selected for their synthetic (polyacrylic acid, PAA), semisynthetic (poly-L-aspartate, PLD; poly-L-glutamate, PLE), or natural (hyaluronate, HA; dextran sulfate, DXS; fucoidan, FUC; alginate, ALG; chondroitin sulfate, CS) origin as well as the inclusion of both carboxylate and sulfate ions.(21-23) These same electrostatic coatings were used to modify polymeric NP cores (polylactide-co-glycolide, PLGA) to test the effects of core composition on NP-cell interactions. We optimized formulations to obtain a diameter of approximately $100 \mathrm{~nm}$ for the liposome and PLGA formulations as the similar sizes would enable cross-core comparisons. We also included commercially manufactured fluorescent carboxylate- and sulfate-modified polystyrene (PS) nanoparticles in a range of diameters from 20-200 nm, enabling study of particle size and surface chemistry. Because of the clinical importance of polyethylene glycol (PEG)-containing 
formulations,(24) PEGylated versions of liposome, PLGA, and PS particles were prepared, including the drug-free versions of two commercial formulations, liposomal doxorubicin

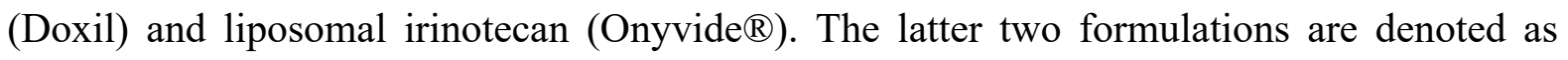
LIPO-5\% PEG* and LIPO-0.3\% PEG*, respectively. All of the nanoparticles examined exhibited negative or neutral net charge, as the focus of this work is on systemic nanoparticle delivery systems. Positively charged nanoparticles have been shown to undergo nonspecific charge interactions with cells and proteins, leading to toxicity and premature clearance in vivo.(25) Dynamic light scattering (DLS) was used to characterize the diameter, zeta potential, and polydispersity index (Figure 1B, Tables S1-S2) of this NP library.

To ensure that our methods led to robust and meaningful data we selected an anti-epidermal growth factor receptor (EGFR) antibody as an active targeting control. A nonlethal EGFR antibody or IgG isotype control was covalently incorporated onto a liposome via a PEG tether.(26)

After incubation with the NP library and fluorescence-activated cell sorting, cells were lysed, and the DNA barcodes were amplified, sequenced, and deconvoluted according to previously detailed protocols. $(14,27)$ After quality control analysis of technical $(n=2)$ and biologic $(n=3)$ replicates, all 488 cell lines met quality control measures and were carried forward for downstream analyses (Figure S2, Supplementary Text).

A probabilistic model was developed and applied to the data to infer the relative distribution of each cell line into the pre-determined bins (A-D) for each NP formulation. The probability of a cell from a given cell line falling into a given bin is used to represent those distributions, i.e., $\mathrm{P}_{\mathrm{A}}+\mathrm{P}_{\mathrm{B}}+\mathrm{P}_{\mathrm{C}}+\mathrm{P}_{\mathrm{D}}=1$ (Figure 1C-D). The technical details and the model's implementation are presented in the Supplementary Text section. Given the concordance of the inferred probabilities among the biologic replicates (Figure S3), we collapsed the replicates through their arithmetic average. Probabilities were then summarized using a weighting factor alpha $(\alpha)$ to calculate a weighted average (WA) for each NP-cell line pair: WA $=-\alpha \mathrm{P}_{\mathrm{A}^{-}}$ $\mathrm{P}_{\mathrm{B}}+\mathrm{P}_{\mathrm{C}}+\alpha \mathrm{P}_{\mathrm{D}}$ in which a higher WA implies higher NP-cell association and vice versa (Figure 1E). We trialed a range of weighting factors $(\alpha=2,10,20$ and 100) and found that downstream results were unchanged with the higher $\alpha$ values (Figure S4), and therefore, $\alpha=2$ was used for subsequent analyses. 

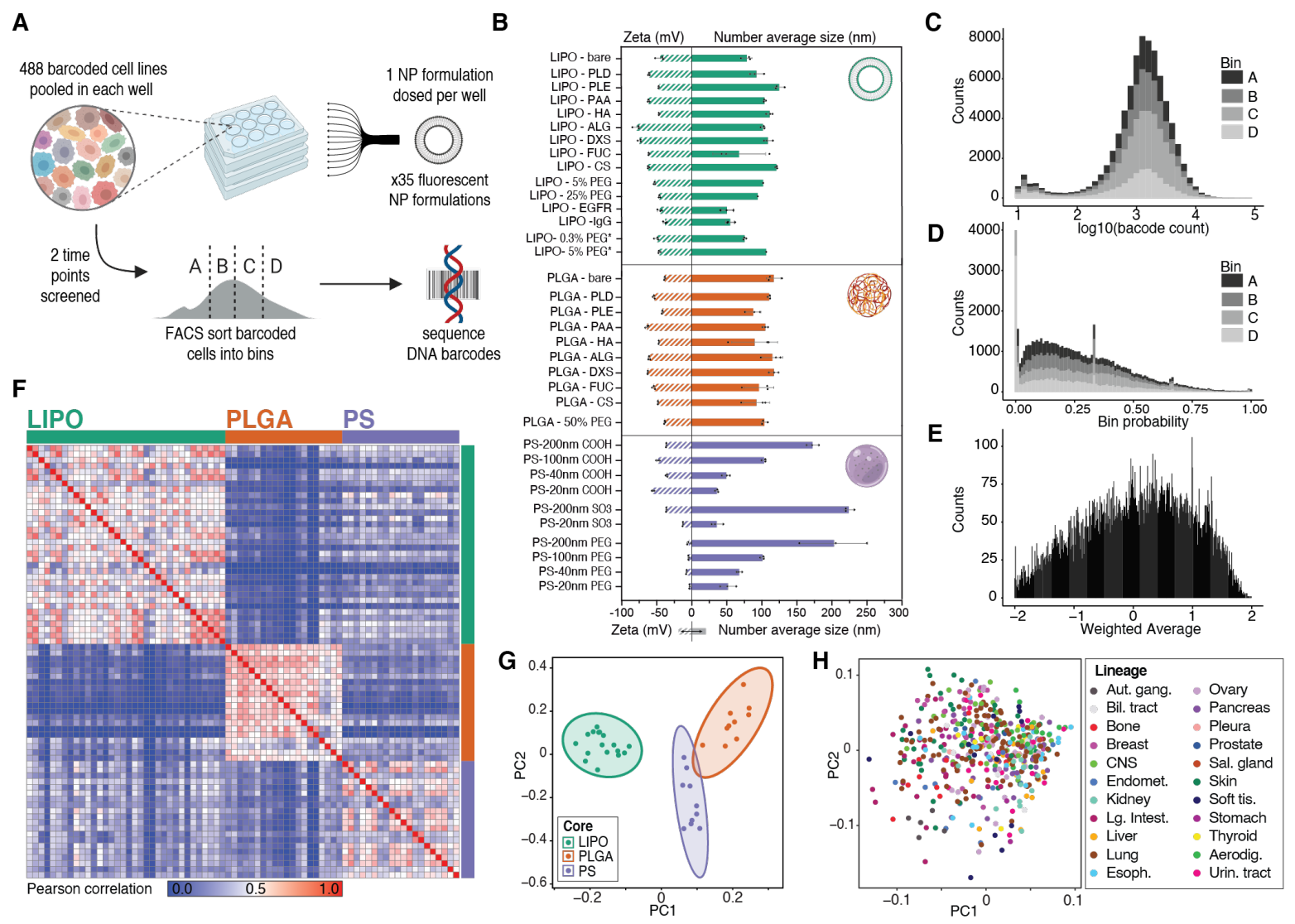

Figure 1. Assessing NP-cell interactions across hundreds of cancer cell lines simultaneously.

(A) Schematic of the nanoPRISM assay: Fluorescently-labeled NPs are incubated with pooled cancer cells before fluorescence-activated cell sorting (FACS) by NP-association and sequencing of DNA barcodes for downstream analyses. (B) Characterization of the diameter and zeta potential of the NP library via dynamic light scattering. Data is represented as the mean and standard deviation of three technical repeats. Formulations marked with an asterisk represent drug-free analogs of clinical liposomal formulations as described in the text. (C) Raw data from the screen was obtained in the form of barcode counts, with similar numerical distribution of barcodes in each bin, represented as a stacked histogram. (D) Accounting for baseline differences in barcode representation yields the probability $(\mathrm{P})$ that each cell line will be found in a particular bin. $(\mathbf{E})$ Probabilities are collapsed into a single weighted average (WA) for each NP-cell line pair. (F) A similarity matrix collapsing WA values for 488 cell lines reveals clusters of NP formulations with the same core formulation. (G-H) Principal component analysis (PCA) of NP-cell line WA values at $24 \mathrm{~h}$ confirms distinct clustering of NP formulations based on core composition but cell lines 
do not form clusters, indicating lineage does not significantly influence NP-cancer cell interactions.

\section{Cancer cells distinguish nanoparticles based on core composition}

Pearson-based unsupervised hierarchical clustering of pairwise WAs identified NP core material as a strong determinant of cell association, with the three core materials tested (liposomal, PLGA and PS) forming distinct clusters (Figure 1F and S5A). This result was unexpected as we hypothesized surface chemistry to be a larger predictor of NP-cell interactions. Principal component analysis (PCA) similarly identified these core specific trends at both the 4 and 24 hour time points (Figures $1 \mathrm{G}$ and S5B). Further analysis within each core material did reveal surface chemistry dependent trends, though they were more subtle than core-based clustering (Figure S6).

In contrast, no clusters were apparent when PCA was performed based on cell line, indicating that cancer cells of the same lineage did not have similar NP-association trends (Figure 1H, Figure S5C). Heterogeneity in NP-cell association in proliferating cells has been attributed to various aspects of cell growth and metabolism.(28-31) To ensure that differential cell proliferation did not confound our results, we performed a parallel growth experiment with the same pooled cells and found no correlation between estimated doubling time and WA (Figure S7).

\section{Cell-intrinsic features mediate nanoparticle trafficking}

We applied data from the Cancer Cell Line Encyclopedia (CCLE)(32, 33) to identify genomic features that act as predictive biomarkers for NP-cell association. To do this, we

employed both univariate analyses and a random forest algorithm to correlate the baseline molecular features of each cell line (cell lineage; gene copy number; messenger RNA, microRNA, protein or metabolite abundance; function-damaging, hotspot or missense mutations) with NP association.

\section{EGFR-targeting compounds identify relevant biomarkers with high confidence}


Using univariate analysis for all CCLE features, we identified EGFR gene expression and protein abundance as the two most significantly correlated hits $\left(\mathrm{q}=4 \times 10^{-100}\right.$ and $\mathrm{q}=4 \times 10^{-76}$, respectively) with anti-EGFR antibody, but much less significantly $\left(\mathrm{q}=6 \times 10^{-9}\right.$ and $\mathrm{q}=4 \mathrm{x}$ $10^{-10}$, respectively) associated with the isotype control (Figure $2 \mathrm{~A}$, top panels).

In EGFR-conjugated liposomes, these same hits were also identified more significantly $\left(\mathrm{q}=6 \times 10^{-21}\right.$ and $\mathrm{q}=2 \times 10^{-18}$, respectively) than the $\operatorname{IgG}$ control $\left(\mathrm{q}=3 \times 10^{-9}\right.$ and $\mathrm{q}=3 \times 10^{-6}$, respectively) (Figure 2A, bottom panels). The statistical significance of EGFR biomarkers was lower for the antibody-conjugated liposome than the free antibody, which may be due to steric blockage introduced by covalently linking an antibody to a NP surface that may interfere with binding to its target.(34) Thus, we demonstrated the ability to quantitatively compare expected biomarker targets of both free antibodies and antibody-conjugated NPs using our platform. This method of analysis will provide therapeutic insights in the design of antibody-drug conjugates, specifically in evaluating the effects of conjugation site or linker chemistry. 
A

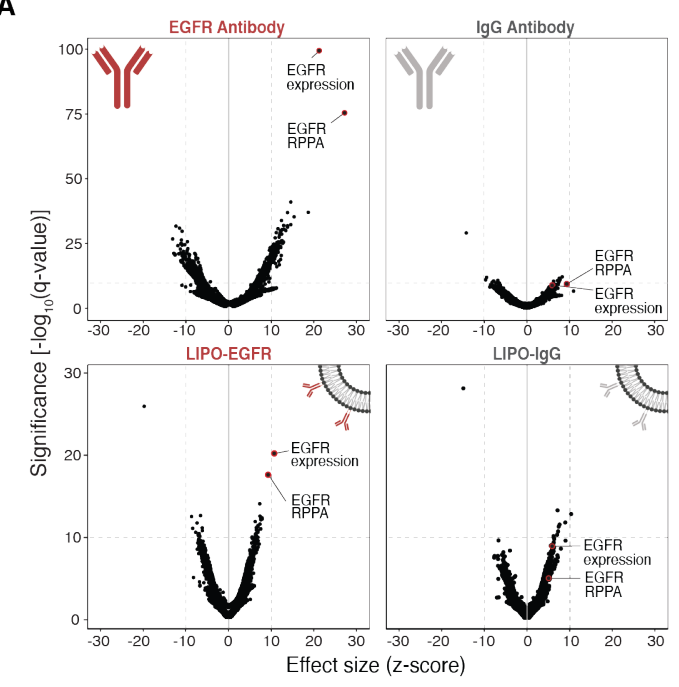

C

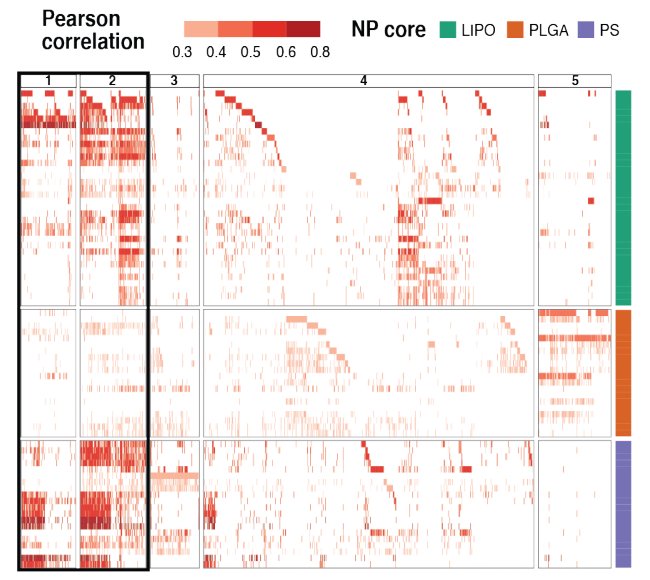

B

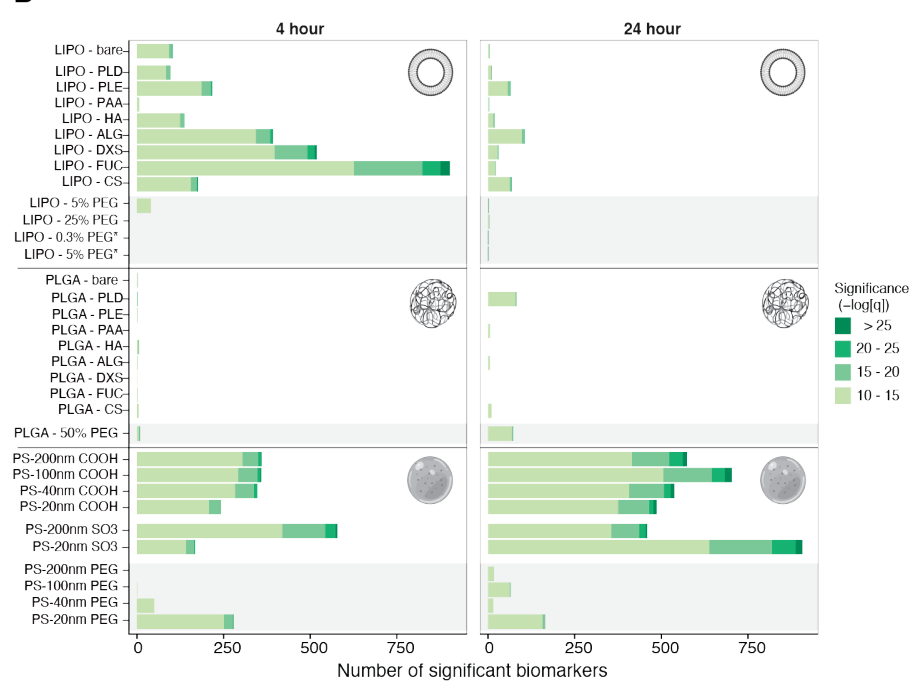

D

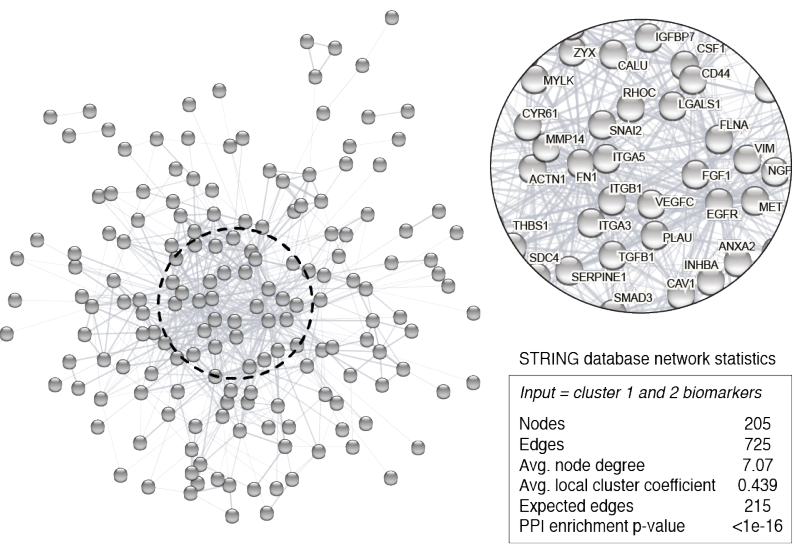

E

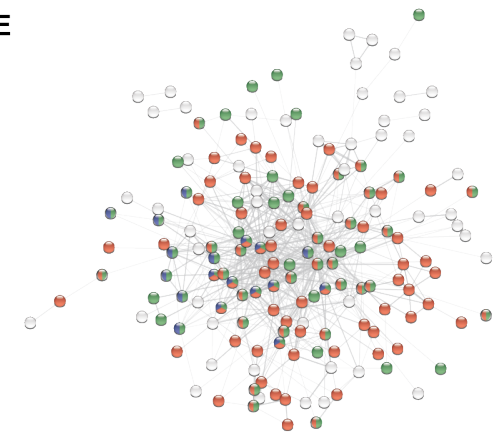

Gene Ontology Cellular Component

G0.0005576 extracellular region $7.710-12$

Go.0005886 plasma membrane $287 \mathrm{e}-09$

G0 0031012 excellular matrix $327 \mathrm{e}-08$

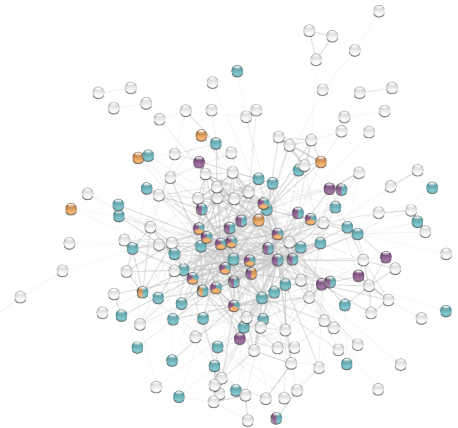

Gene Ontology Biological Process $\quad$ FDR G0.0009653 anat. struct. morphogenesis 6.760-16 G0-0010810 reg. cell-substrate adhesion $1.94 \mathrm{e}-12$ GO 0030335 pos regulation cell motility 1.94e-12

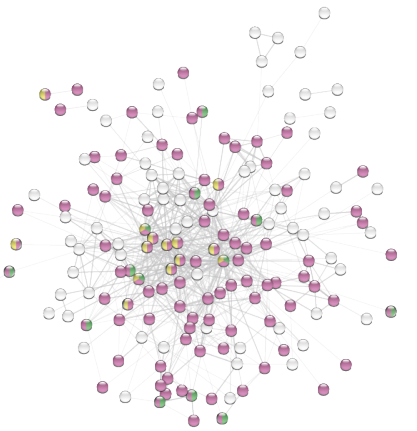

Gene Ontology Molecular Function $\quad$ FDR G0:0019838 growth factor binding $1.200-06$ G0.0005515 protein binding $1.20 \mathrm{e}-06$

G0 0050839 cell adhes mol binding $1.43 e-06$

Figure 2. Correlative genomic analysis identifies expected validation biomarkers as well as hundreds of formulation- and time-dependent biomarkers. (A) Univariate analysis reveals EGFR gene expression and protein abundance (via reverse phase protein array; RPPA) to be strongly and positively correlated with high anti-EGFR association (top left). EGFR-related 
markers are much less significant in the isotype control (top right). The same EGFR-related hits, in addition to NP specific markers, are observed for antibody-conjugated liposomes (bottom row). (B) Univariate analysis identifies genomic features correlated with NP association. All biomarkers meeting a significance threshold of $-\log 10(q-v a l u e)>10$ are shown as stacked bar graphs separated by NP formulation and time point. PEGylated NP formulations are highlighted with a gray background. (C) A heatmap showing all gene- and protein- expression features with positive correlation identified by random forest algorithm in columns, and NP formulations in rows. Features are colored based on their Pearson correlation and clustered using k-means clustering, with clusters $1+2$ highlighted as features present across multiple NP formulations. (D) Visual representation of the STRING network generated by inputting the 205 features from clusters $1+2$, with network statistics. Each node represents a feature, and the edges represent predicted functional associations. The most interconnected nodes are labeled in the zoomed inset. (E) Top gene ontology enrichments and false discovery rates associated with the network presented in panel (D).

\section{Biomarker number and significance are influenced by nanoparticle properties}

We employed univariate analysis to correlate association and CCLE features for each NP formulation, thresholding q-values less than $1 \times 10^{-10}$ (Figure $2 \mathrm{~B}$ ). Selection of this cutoff was guided by the IgG-conjugated antibody analysis, which returned few hits above this threshold. For liposomal NPs, we observed that the number of significant biomarkers was higher at $4 \mathrm{~h}$ than $24 \mathrm{~h}$. We believe this may be indicative of active uptake processes, established to take place within the first few hours of NP-cell interactions, whereas at 24 hours, we may be capturing features associated with less specific interactions. $(35,36) \mathrm{We}$ also observed that liposome surface modification influences the number and significance of biomarkers. Specifically, liposomes electrostatically coated with polysaccharides (HA, ALG, DXS, FUC, CS) had the highest amount of associated biomarkers, which we hypothesize is due to the high degree of interactions between sugars and cell surface proteins as well as the potential for naturally occurring polysaccharides to interact with a wide range of cell surface elements.(22, 37, 38) In line with this hypothesis, the addition of PEG, a well-established antifouling polymer, reduces the number and significance of associated biomarkers almost to zero. In 
contrast to the highly specific hits generated from EGFR-conjugated liposomes (formulated using 25\% PEG liposomes), this abrupt decrease in significant biomarkers further indicates the ability of our platform to identify specific NP binding and recognition elements. In contrast to the liposomal formulations, PLGA formulations, regardless of surface modification, resulted in few biomarkers at either time point. Lastly, a high number of significant biomarkers was associated with both carboxylated and sulfated PS NPs included in our screen, though there was no time dependence, in contrast to the liposomal formulation. While this result was initially surprising, as the PS formulations are made of synthetic polystyrene polymers, meaningful biological interactions with anionic polystyrenes, both in polymer and particle form, have been reported.(39) Specifically, it was described that these systems have the appropriate mix of hydrophobicity and anionic charge character to interact favorably with trafficking proteins, including the caveolins.

\section{NP biomarkers are connected and create trafficking networks}

We additionally identified predictive biomarkers for the tested NP formulations using a random-forest algorithm (methods in Supplementary Text). We annotated these candidate biomarkers to include the categories of gene expression, gene copy number, or protein abundance. Data from the $4 \mathrm{~h}$ time point was chosen for this analysis based on the EGFRrelated hits for liposomes, which were more significant at $4 \mathrm{~h}$ than at $24 \mathrm{~h}$. As we were interested in applying this approach to identify cellular features positively correlated with uptake (e.g., increased expression of trafficking proteins), hits negatively correlated with NP association were removed from this analysis. Next, we used K-means clustering to visualize biomarkers based on their relative importance and presence across formulations (Figure 2C). Clusters 1 and 2 contained many hits shared across NP formulations and were especially enriched for liposomal and PS NPs. These 205 genes and proteins were input into the STRING database (40-42) to generate a protein-protein interaction (PPI) network that was found to be highly interconnected (PPI enrichment $\mathrm{p}$-value $<1 \times 10^{-16}$ ) (Figure 2D). Notably, the network is enriched in proteins found in the plasma membrane, extracellular region, and extracellular matrix (false discovery rate $[\mathrm{FDR}]=8 \times 10^{-12}, 3 \times 10^{-9}$, and $3 \times 10^{-8}$, respectively) based on enrichment analysis with gene ontology (GO) localization datasets (Figure 2E).(43-45) The identification of overlapping biomarkers that are localized to the cell surface and have 
established protein-protein interactions led us to hypothesize that these proteins are important in early NP trafficking. Enrichment analyses using GO molecular functions datasets showed enrichment in numerous binding processes (Data S1, Figure 2E), giving further credence to this theory. These results serve as a framework for the comprehensive investigation of cellular processes important for NP engagement, which may prove useful for fundamental trafficking studies and target identification.

\section{SLC46A3 is a negative regulator of liposomal NP uptake}

A
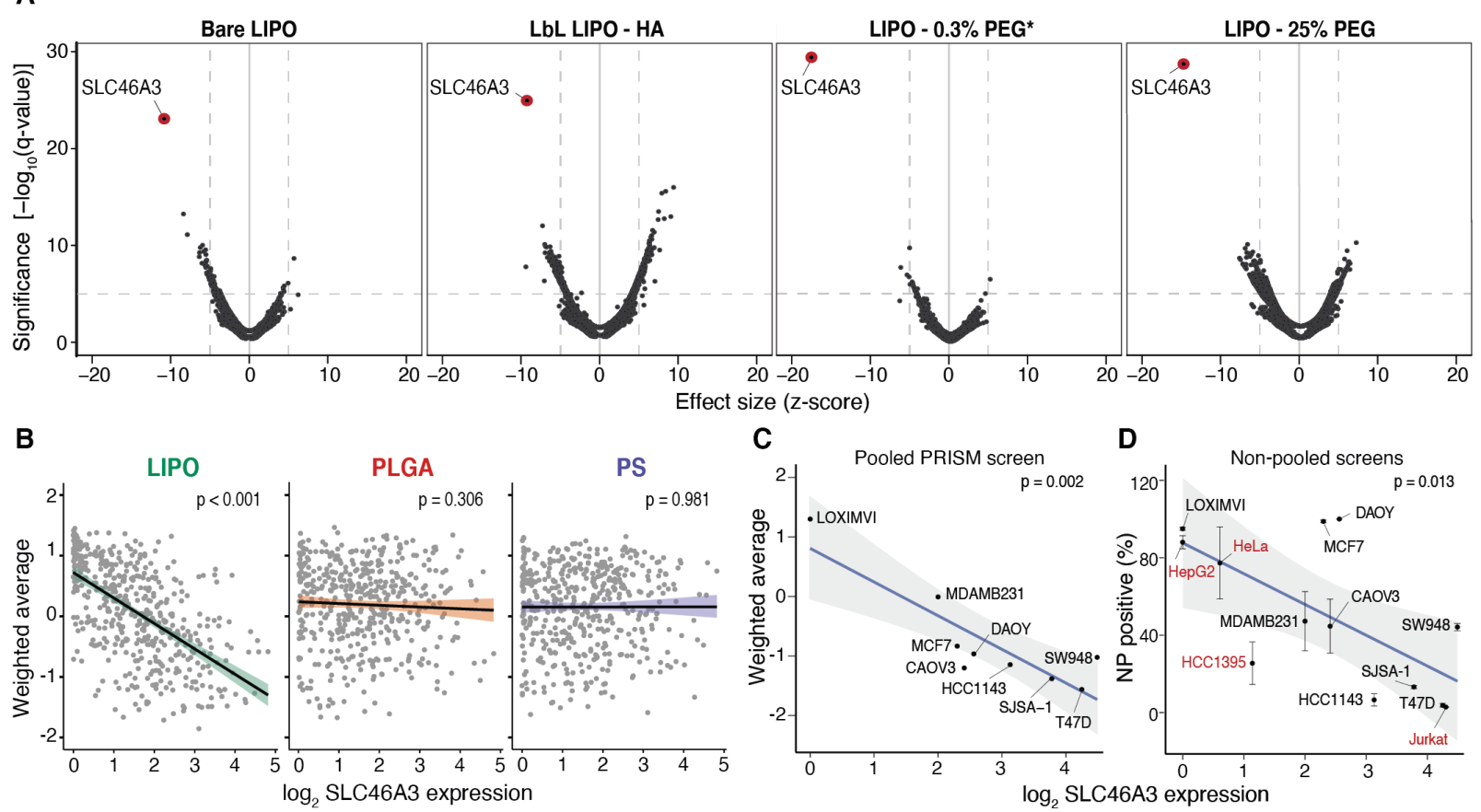

Figure 3. Native expression of the lysosomal transporter SLC46A3 is strongly predictive of NP-cell interaction for liposome formulations. (A) Univariate analysis identifies SLC46A3 expression as strongly yet inversely correlated with liposome association, regardless of liposomal surface modification. (B) Using linear regression to evaluate the biomarker relationship across core formulations reveals SLC46A3 expression is inversely correlated with NP association in liposome-cell line pairs $(p<0.001)$ but not PLGA- and PS-cell line pairs $(p>0.05) ; \mathrm{n}=488$ for each plot. (C) Cell lines in the nanoPRISM pool exhibit a range of natural SLC46A3 expression levels with a log linear correlation with uptake of liposomes. (D) This correlation is also exhibited when assessing liposome-cell associations via flow cytometry in a non-pooled fashion $(p=$ 
0.013). Cell lines in red were not part of the pooled PRISM screen. Data represented in D is shown as the mean and standard deviation of four biological replicates.

Evaluating univariate results across NP formulations, we identified one biomarker with a strong, inverse relationship with liposomal NP association: expression of solute carrier family 46 member 3 (SLC46A3), a lysosomal transporter. SLC46A3 has been implicated in the metabolism of antibody-drug conjugates,(46-48) but this is, to our knowledge, the first description of SLC46A3 as a NP-specific biomarker. Moreover, downregulation of SLC46A3 has been reported to play a role in cancer cell malignancy and therapy resistance in several cancers. $(49,50)$ Given these significant therapeutic implications, we sought to validate the predictive power of SLC46A3 expression for liposomal NP association.

SLC46A3 expression was the most significant hit on univariate analysis and also the top ranked random forest feature for each liposomal NP tested at $24 \mathrm{~h}$, regardless of surface modification (Figures 3A and S8). This inverse relationship between SLC46A3 expression and NP association was found to be specific to liposomal NPs, and not observed with PLGA or PS NPs, and was maintained regardless of cancer cell lineage (Figures 3B and S8).

We selected nine cancer cell lines from the nanoPRISM pool and four additional cell lines, spanning multiple lineages, with a range of native $S L C 46 A 3$ expression levels for screening in a non-pooled fashion (Figure 3C-D). Analogous to the pooled screen, individual cell lines were profiled using flow cytometry and NP-associated fluorescence was quantified after $24 \mathrm{~h}$ incubation (Figures 3D and S9). In line with observations from pooled screening, the inverse relationship between liposome association and native $S L C 46 A 3$ expression was maintained, suggesting that SLC46A3 may play a key role in trafficking of liposomal NPs.

To further functionally probe whether SLC46A3 expression level governs NP association, we selected two cancer cell lines from the pooled screen that displayed strong phenotypes (Figure 4A). The melanoma cell line LOXIMVI exhibited high association and breast cancer cell line T47D exhibited low association with liposomal NP formulations (Figure 4B). We developed a toolkit using these cell lines by knocking out SLC46A3 in T47D cells and inducing overexpression in LOXIMVIs (Figures S10A-H).

As SLC46A3 is a protein associated with lysosomal membranes(46-48), we utilized LysoTracker dye to evaluate the effect of SLC46A3 modulation on endolysosomal 
compartments in both T47D and LOXIMVI engineered cell lines (Figure 4C). We observed an SLC46A3 expression level-dependent change: cells with higher SLC46A3 levels (T47Dvector control, LOXIMVI-SLC46A3 OE) exhibited more brightly dyed endolysosomal compartments compared to their low SLC46A3 expression counterparts (T47D-SLC46A3 knockout, LOXIMVI-vector control).

Overexpression of SLC46A3 in LOXIMVI cells significantly abrogated interaction with bare liposomes $(p=0.006)$ using flow cytometry profiling (Figure 4D). The T47D-SLC46A3 knockout cell line demonstrated significantly increased association with bare liposomes compared to parental or vector control lines $(p=0.0017$, Figure 4D). We further confirmed that these trends are generalizable across a range of surface functionalized liposomes (Figure 4E). Moreover, no significant changes in NP association were observed for PLGA and PS NPs. We also confirmed that the presence of serum proteins in cell culture media does not abrogate this trend (Figure S10I). Taken together, these data indicate modulation of SLC46A3 alone in cancer cells is sufficient to negatively regulate association and uptake of liposomal NPs. 
A

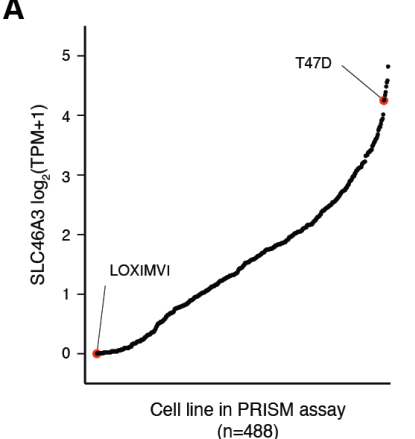

D

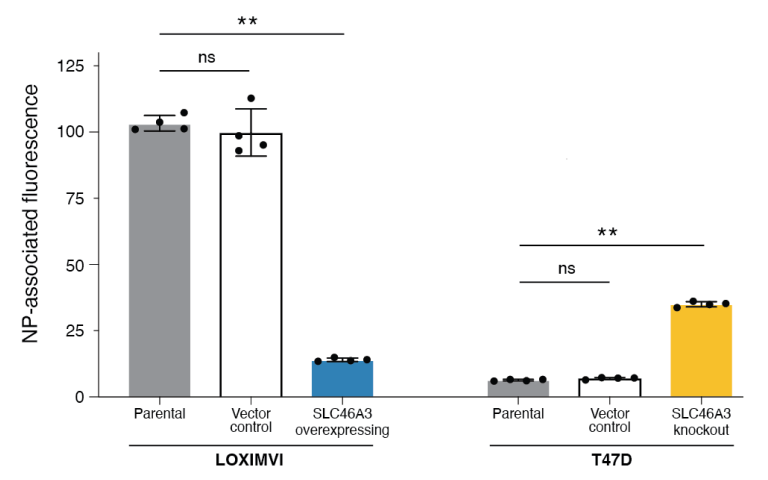

B

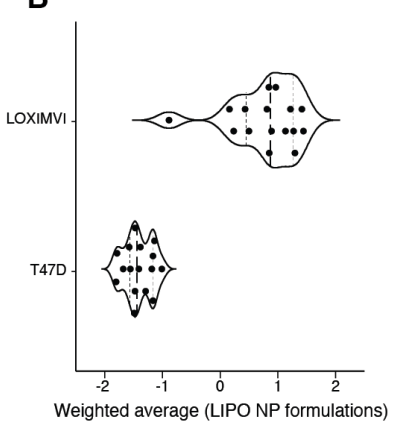

C
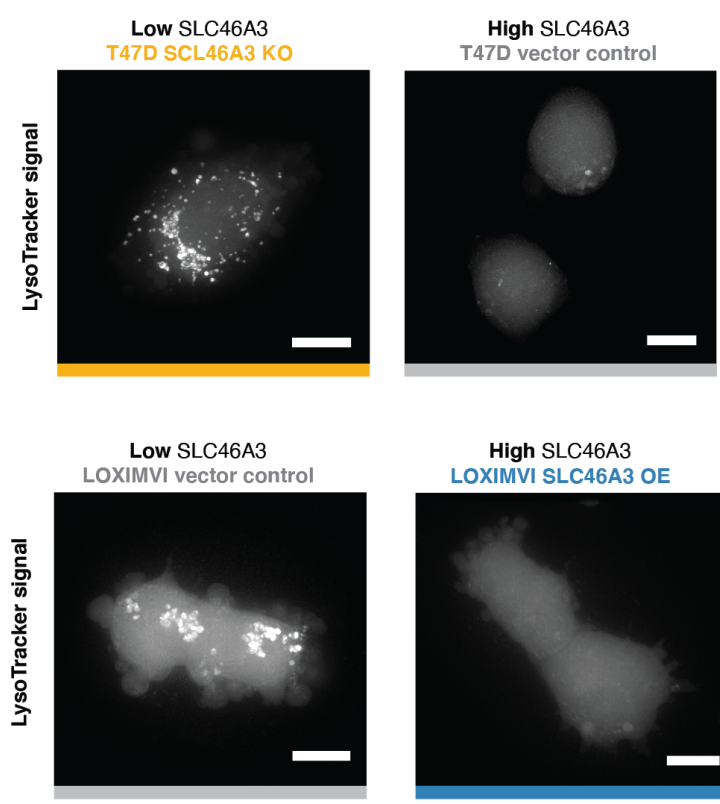

High SLC46A3 LOXIMVI SLC46A3 OE

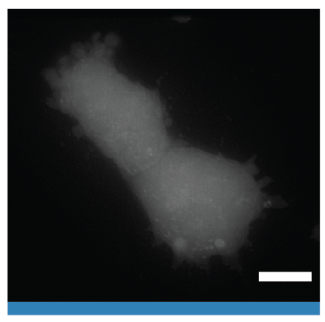

$\mathbf{E}$
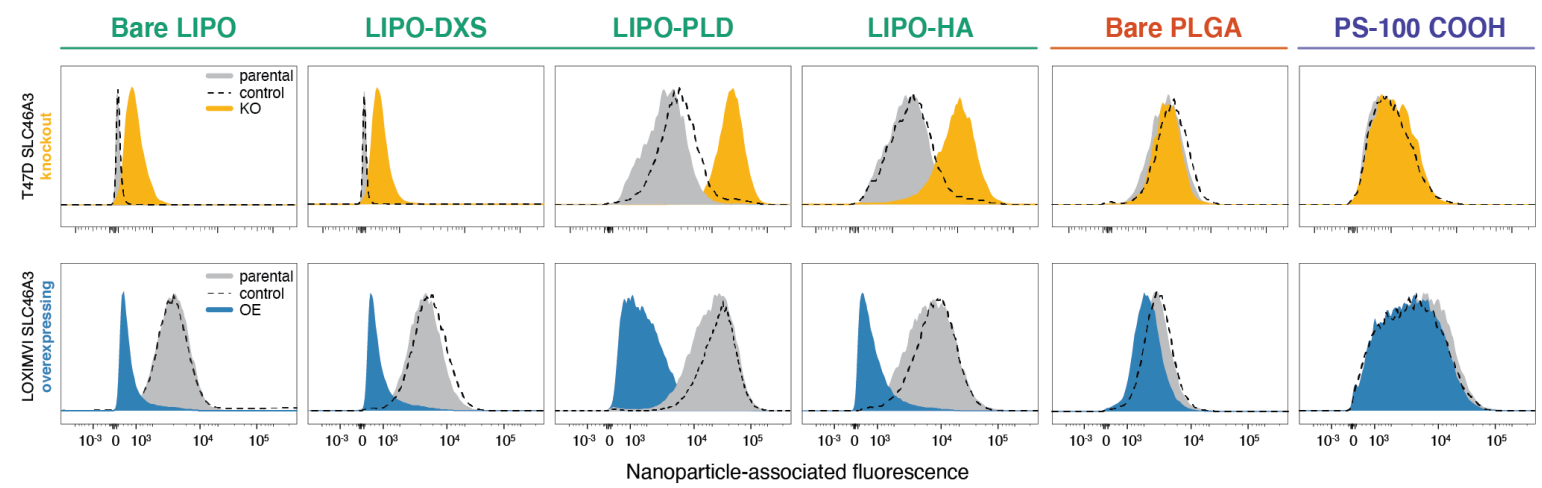

Figure 4. Modulating $S L C 46 A 3$ expression in cancer cell lines is sufficient to negatively regulate interaction with liposome NP formulations. (A) T47D and LOXIMVI cells have high and low $S L C 46 A 3$ expression, respectively, with respect to $S L C 46 A 3$ expression levels represented in the nanoPRISM cell line pool. (B) LOXIMVI cells have predominantly high association with all tested liposomal NP formulations and T47D cells have low association with all tested liposomal NP formulations. (C) Representative micrographs of Lysotracker signal in engineered cell lines showing endolysosomal compartments. Scale bars $=10 \mu \mathrm{m}$. (D) Using lentivirus to overexpress SLC46A3 in LOXIMVI cells and CRISPR/Cas9 to knock out SLC46A3 in T47D cells, we show that modulation results in significantly changed liposome association, as determined via flow cytometry (** $p<0.001$, Kruskal-Wallis test), NP-associated fluorescence is defined as median 
fluorescence intensity normalized to untreated cells. Data is represented as the mean and standard deviation of four biological replicates. (E) These shifts in NP association were consistently observed across all tested liposomes, independent of surface modification. No shifts were observed with PLGA or PS formulations.

As flow cytometry does not provide spatial information with respect to NP-cell interactions, we employed imaging cytometry to characterize NP localization in a high throughput manner (Figure 5A-F). We selected four representative formulations: three liposomal NPs to probe the relationship of SLC46A3 expression with liposome trafficking; and one PLGA NP formulation with a common outer layer.

Consistent with trends observed by flow cytometry, we observed an inverse relationship between NP intensity and SLC46A3 expression for liposomal, but not PLGA, NPs (Fig 5A-B, S11). Using brightfield images, we applied a mask to investigate cellular localization of NPs. All tested formulations were internalized, and this did not change with SLC46A3 modulation (Figure 5C-D), so we hypothesize that SLC46A3 plays a role in retention of NPs rather than directly blocking uptake.

We investigated localization of NPs by scoring NP signal based on distribution within each cell (Figure 5E-F, Figure S11D). We observed stark differences in median cellular distribution scores of liposomal NPs in relation to SLC46A3 expression levels in T47D cells. This was not observed for PLGA NPs, mimicking the previously observed core-specific relationship between NP-cell association and SLC46A3 expression. Changes in this score, though less pronounced, were also observed for liposomal NPs in LOXIMVI cells.

To confirm these findings with higher spatial resolution, we employed deconvolution microscopy of live cells and incorporated a lysosomal stain to observe changes in intracellular trafficking (Figure 5G-H). NPs appeared uniformly distributed within T47D-SLC46A3 KO cells, co-localizing with endolysosomal vesicles. In contrast, LIPO-PLD NPs were localized to large endolysosomal clusters in T47D-vector control cells. This trend was also observed for LIPO-PLE and LIPO-0.3\% PEG* NPs and at the earlier time point of $4 \mathrm{~h}$ (Figure S12). Changes in localization were not observed for the tested PLGA PLD NPs. This again indicates a NP core-dependent relationship with SLC46A3. 
In the engineered LOXIMVI cell lines, we also observed co-localization of liposomal NPs with endolysomal signal. However, predictable changes in NP localization were not detected, in line with smaller changes in median cellular distribution scores. Taken together, these results suggest that the relationship between SLC46A3 expression and NP uptake is twofold: SLC46A3 predictably and negatively regulates the amount of liposomal NPs accumulating within a cell and modulates subcellular trafficking in certain contexts. 
A

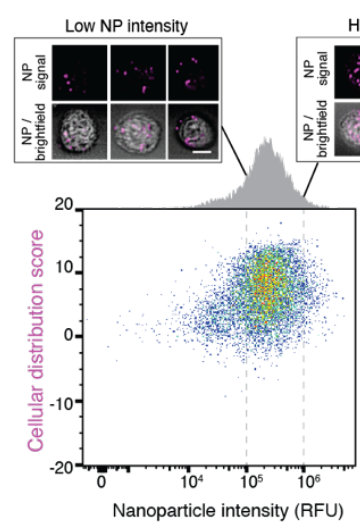

B

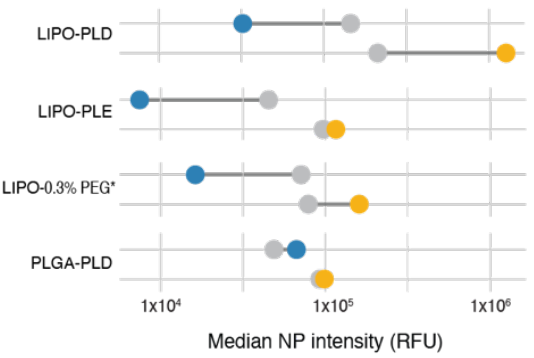

C

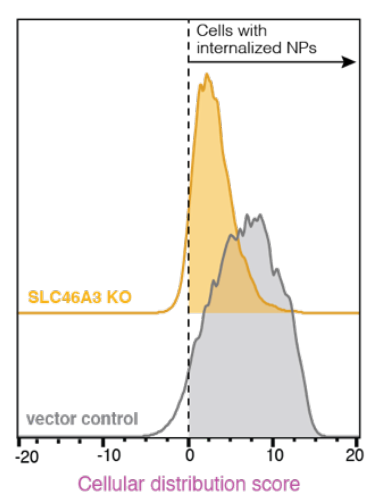

D

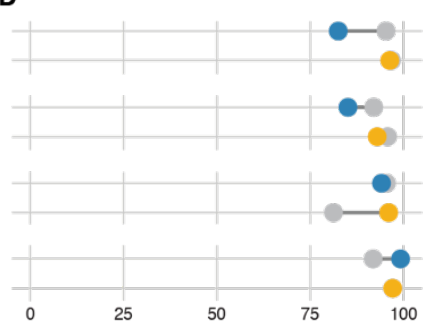

E

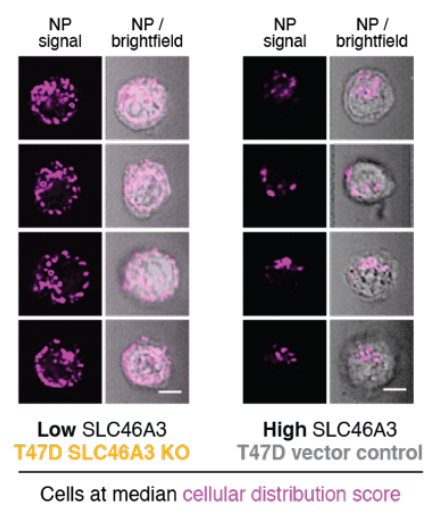

$\mathbf{F}$

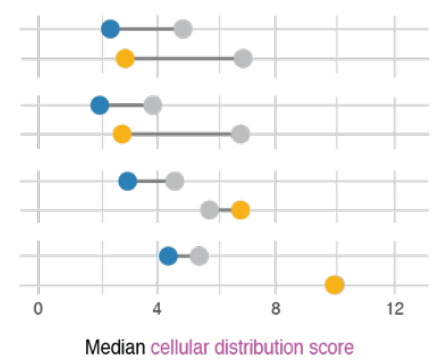

Median cellular distribution score

\section{G}
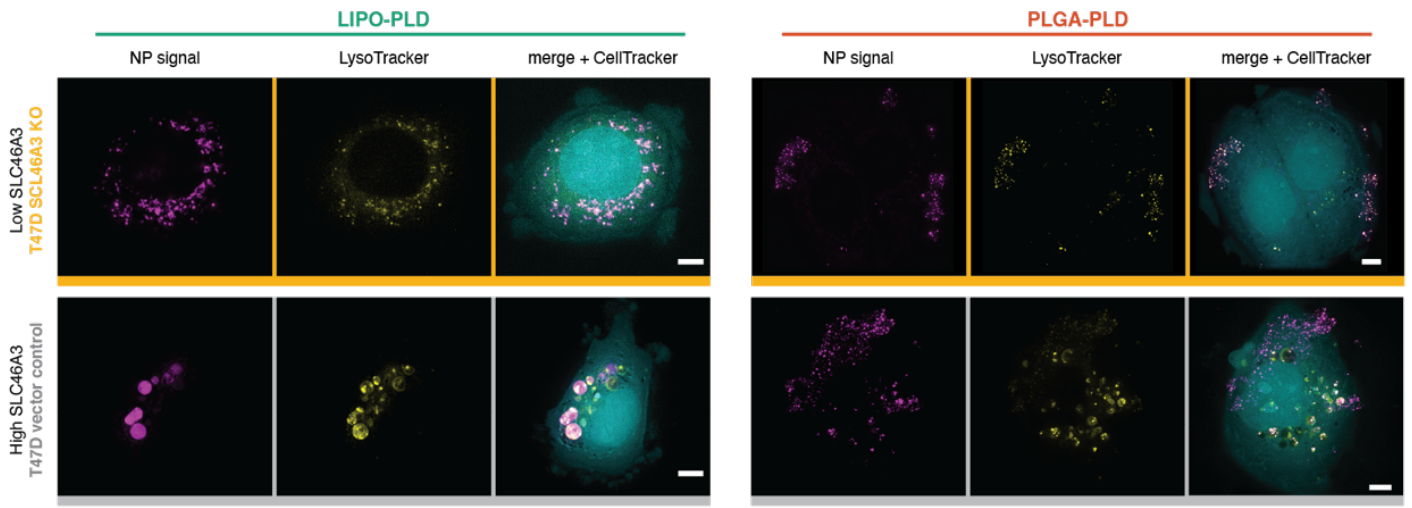

H
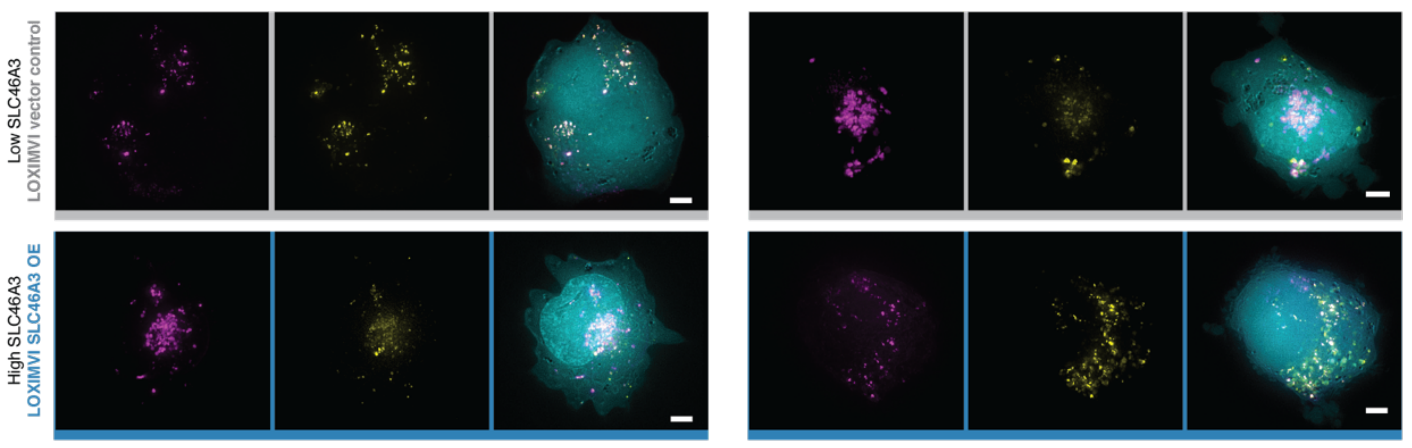


\section{Figure 5. High throughput imaging cytometry confirms NP internalization and reveals} SLC46A3 dependent changes to intracellular trafficking. (A) Imaging cytometry was used to investigate the intensity (x-axis) and distribution (y-axis) of NPs in a high-throughput manner. Bivariate density plot of $n=10,000$ cells (T47D-vector control) after $24 \mathrm{~h}$ incubation with LIPOPLD NPs, with representative cell images at low and high NP signal. (B) Cellular distribution patterns of NPs were scored such that scores greater than 0 indicate cells with internalized NPs. Representative data from LIPO-PLD NPs in engineered T47D cells are shown. (C) Representative cell images at the median cellular distribution score for engineered T47D cells treated with LIPOPLD NPs. (D) Quantification of median intensity of tested NP formulations in engineered T47D and LOXIMVI cell lines demonstrated SLC46A3-dependent changes. (E) NPs remained predominantly internalized independent of SLC46A3 expression levels. (F) Shifts in the median cellular distribution scores were observed in response to SLC46A3 modulation. Live cell micrographs of (G) T47D-vector control and T47D-SLC46A3 knockout cells and (H) LOXIMVIvector control and LOXIMVI-SLC46A3 OE cells incubated with LIPO-PLD and PLGA-PLD NPs for 24h. NP signal is pseudo-colored magenta, LysoTracker signal yellow, and CellTracker cyan. Scale bar $=5 \mu \mathrm{m}$.

\section{Liposome retention and accumulation remains $S L C 46 A 3-d e p e n d e n t$ in vivo}

To evaluate the potential clinical utility of SLC46A3 as a negative regulator of liposomal NP delivery, we tested in vivo delivery of an FDA-approved nanoparticle analog, the drug-free version of liposomal irinotecan (LIPO-0.3\% PEG*), in mice bearing subcutaneous LOXIMVI flank tumors. NPs were administered via a one-time intratumoral (IT) injection or repeat intravenous (IV) administration to evaluate tumor retention and accumulation, respectively (Figure 6A, Figure S13).

NP signal was quantified both 4 and $24 \mathrm{~h}$ following IT administration. In line with our hypothesis, as well as in vitro NP-associated fluorescence data (Figure S13A), we observed an inverse relationship between SLC46A3 expression and LIPO-0.3\% PEG* NP retention that became more pronounced over time ( $p=0.0115,4 \mathrm{~h} ; p=0.0002,24 \mathrm{~h}$ ) (Figure 6C-D, Figure S13B-E). Moreover, these findings also align with our initial nanoPRISM findings, in which 
SLC46A3 expression was a more significant biomarker at $24 \mathrm{~h}$ (q-value $=3.49 \times 10^{-30}$, Table S2, Figure S8) than at $4 \mathrm{~h}\left(\mathrm{q}-\mathrm{value}=1.47 \times 10^{-4}\right.$, Table S2, Figure S8).

To determine if this newly identified biomarker can be used to predictably govern accumulation of nontargeted NPs, which bear no specific functional ligands on their surface, following systemic administration, we quantified NP signal following IV injections. Notably, we observed a significant relationship between SLC46A3 and NP accumulation $(p=0.0019)$ (Figure $6 \mathrm{D}$, Figure S13F). This demonstrates the predictive power of SLC46A3 as a NP specific biomarker that holds true even in complex physiologic settings.

Together, these data highlight the real-world relevance of the nanoPRISM screening assay in general as well as the utility of SLC46A3 in particular a clinically actionable biomarker.

A

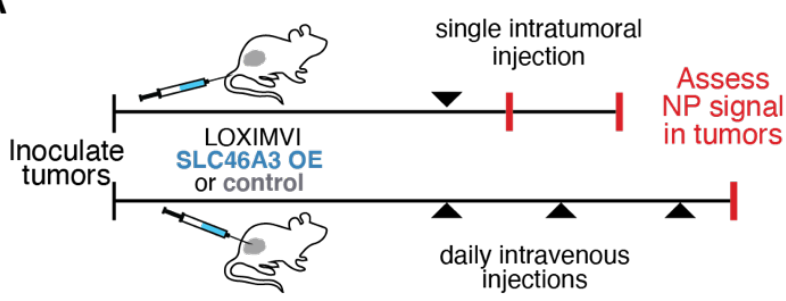

B

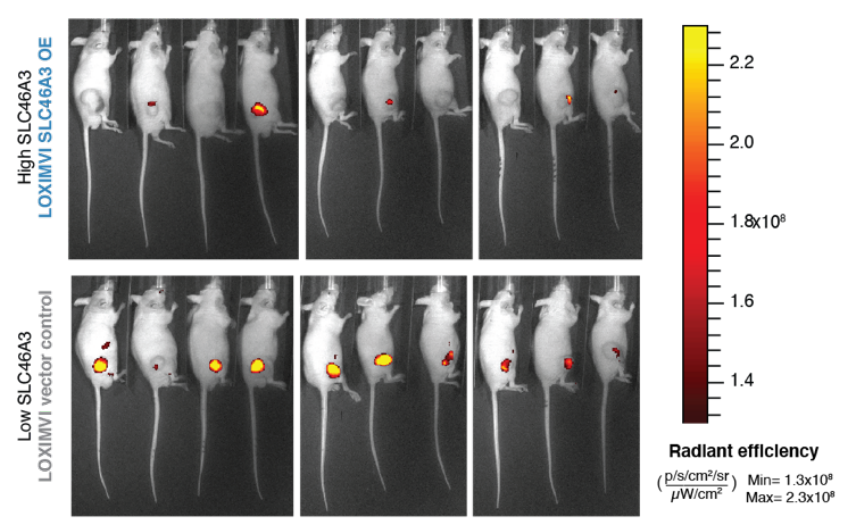

C

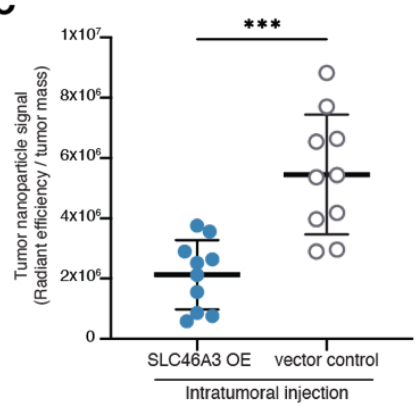

D

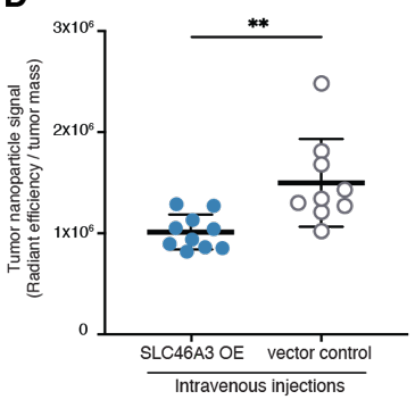


Figure 6. Retention and accumulation of PEGylated liposomes (LIPO-0.3\% PEG*) in LOXIMVI tumors is dependent on SLC46A3 expression. (A) Fluorescently labeled LIPO-0.3\% PEG* NP were administered to mice bearing LOXIMVI flank tumors via a one-time intratumoral injection or repeat intravenous injections. (B) Whole animal fluorescence images of mice (4 males, 6 females per group) $24 \mathrm{~h}$ after being intratumorally injected with LIPO-0.3\% PEG* NPs. (C) Quantification of LIPO-0.3\% PEG* NP retention after intratumoral administration to LOXIMVI flank tumors. (D) Quantification of LIPO-0.3\% PEG* NP accumulation after repeat IV injections. In panels C-D, nanoparticle signal is expressed on the y-axis as radiant efficiency divided by tumor mass (mg). The mean and standard deviation of $n=10$ are shown with the exception of the LOXIMVI-vector control, repeat IV injection group, where $\mathrm{n}=9(* *<0.01$, $* * *<0.001$, MannWhitney test).

\section{Discussion and Conclusions}

This work represents the first high-throughput interrogation of NP-cancer cell interactions through the lens of multi-omics. Harnessing the power of pooled screening and high throughput sequencing, we developed and validated a platform to identify predictive biomarkers for NP interactions with cancer cells. We utilized this platform to screen a 35 member NP library against a panel of 488 cancer cell lines. This enabled the comprehensive study and identification of key parameters mediating NP-cell interactions, highlighting the importance of considering both nanomaterials and cellular features in concert.

Moreover, through the use of univariate analyses and random forest algorithms, we identified biomarkers correlated with NP association. The robust and quantitative nature with which we detected EGFR hits for antibody-targeted NPs shows the utility of this platform for the development and optimization of antibody-targeted therapeutics.

By clustering NP-specific biomarkers across formulations, we constructed interaction networks, identifying and connecting genes associated with NP binding, recognition, and subcellular trafficking. This provides the scientific community with a blueprint for the fundamental study of cellular processes mediating NP engagement, with applications for both basic and translational research.

We additionally identified gene expression of $S L C 46 A 3$ as a negative regulator of liposomal NP uptake. We validated these findings in a panel of non-pooled cell lines and 
engineered cell lines with modulated SLC46A3 levels. Importantly, as all current FDA approved NPs for anticancer applications are liposomal formulations, there is significant potential for this biomarker to be quickly implemented in clinical studies with existing, approved formulations. To this end, we recapitulated our findings in an in vivo model using an analog of an FDA-approved liposomal NP formulation. Our findings present the key first step toward identifying and utilizing such NP-specific biomarkers.

In summary, we present a powerful platform to study NP-cancer cell interactions simultaneously through the use of pooled screening, genomics, and machine learning algorithms. This provides a new dimension to the study of cancer nanomedicine. Application of this platform will serve useful not only for the rational design of nanocarriers, but also for the identification of specific phenotypes primed to benefit from targeted drug delivery and nanomedicine. 


\section{References and Notes}

1. J. J. Shi, P. W. Kantoff, R. Wooster, O. C. Farokhzad, Cancer nanomedicine: progress, challenges and opportunities. Nat. Rev. Cancer 17, 20-37 (2017).

2. M. J. Mitchell, M. M. Billingsley, R. M. Haley, M. E. Wechsler, N. A. Peppas, R. Langer, Engineering precision nanoparticles for drug delivery. Nat. Rev. Drug. Discov. 20, 101-124 (2020).

3. S. Tran, P. J. DeGiovanni, B. Piel, P. Rai, Cancer nanomedicine: a review of recent success in drug delivery. Clin. Transl. Med. 6, 1-21 (2017).

4. S. Wilhelm, A. J. Tavares, Q. Dai, S. Ohta, J. Audet, H. F. Dvorak, W. C. W. Chan, Analysis of nanoparticle delivery to tumours. Nat. Rev. Mater. 1, 16014 (2016).

5. Y. H. Cheng, C. L. He, J. E. Riviere, N. A. Monteiro-Riviere, Z. M. Lin, Meta-Analysis of Nanoparticle Delivery to Tumors Using a Physiologically Based Pharmacokinetic Modeling and Simulation Approach. ACS Nano 14, 3075-3095 (2020).

6. Y. S. Youn, Y. H. Bae, Perspectives on the past, present, and future of cancer nanomedicine. Adv. Drug Deliver. Rev. 130, 3-11 (2018).

7. W. Poon, B. R. Kingston, B. Ouyang, W. Ngo, W. C. W. Chan, A framework for designing delivery systems. Nat. Nanotechnol. 15, 819-829 (2020).

8. W. Poon, Y. N. Zhang, B. Ouyang, B. R. Kingston, J. L. Y. Wu, S. Wilhelm, W. C. W. Chan, Elimination Pathways of Nanoparticles. ACS Nano 13, 5785-5798 (2019).

9. S. Correa et al., Tuning Nanoparticle Interactions with Ovarian Cancer through Layer-byLayer Modification of Surface Chemistry. ACS Nano 14, 2224-2237 (2020).

10. N. Boehnke, S. Correa, L. Hao, W. Wang, J. P. Straehla, S. N. Bhatia, P. T. Hammond, Theranostic Layer-by-Layer Nanoparticles for Simultaneous Tumor Detection and Gene Silencing. Angew. Chem. Int. Ed. Engl. 59, 2776-2783 (2020).

11. N. Boehnke, K. J. Dolph, V. M. Juarez, J. M. Lanoha, P. T. Hammond, Electrostatic Conjugation of Nanoparticle Surfaces with Functional Peptide Motifs. Bioconjug. Chem. 31, 2211-2219 (2020).

12. J. E. Dahlman et al., Barcoded nanoparticles for high throughput in vivo discovery of targeted therapeutics. Proc. Natl. Acad. Sci. U.S.A. 114, 2060-2065 (2017).

13. B. Nogrady, How cancer genomics is transforming diagnosis and treatment. Nature 579, S10-S11 (2020). 
14. C. N. Yu et al., High-throughput identification of genotype-specific cancer vulnerabilities in mixtures of barcoded tumor cell lines. Nat. Biotechnol. 34, 419-423 (2016).

15. S. M. Corsello et al., Discovering the anticancer potential of non-oncology drugs by systematic viability profiling. Nat. Cancer 1, 235-248 (2020).

16. S. Correa, N. Boehnke, E. Deiss-Yehiely, P. T. Hammond, Solution Conditions Tune and Optimize Loading of Therapeutic Polyelectrolytes into Layer-by-Layer Functionalized Liposomes. ACS Nano 13, 5623-5634 (2019).

17. S. Correa et al., Highly Scalable, Closed-Loop Synthesis of Drug-Loaded, Layer-byLayer Nanoparticles. Adv. Funct. Mater. 26, 991-1003 (2016).

18. Z. J. Deng, S. W. Morton, E. Ben-Akiva, E. C. Dreaden, K. E. Shopsowitz, P. T. Hammond, Layer-by-Layer Nanoparticles for Systemic Codelivery of an Anticancer Drug and siRNA for Potential Triple-Negative Breast Cancer Treatment. ACS Nano 7, 9571-9584 (2013).

19. S. W. Morton, Z. Y. Poon, P. T. Hammond, The architecture and biological performance of drug-loaded LbL nanoparticles. Biomaterials 34, 5328-5335 (2013).

20. G. Decher, Fuzzy nanoassemblies: Toward layered polymeric multicomposites. Science 277, 1232-1237 (1997).

21. E. C. Dreaden et al., Tumor-Targeted Synergistic Blockade of MAPK and PI3K from a Layer-by-Layer Nanoparticle. Clin. Cancer Res. 21, 4410-4419 (2015).

22. E. C. Dreaden, S. W. Morton, K. E. Shopsowitz, J. H. Choi, Z. J. Deng, N. J. Cho, P. T. Hammond, Bimodal Tumor-Targeting from Microenvironment Responsive Hyaluronan Layer-by-Layer (LbL) Nanoparticles. ACS Nano 8, 8374-8382 (2014).

23. O. P. Oommen, C. Duehrkop, B. Nilsson, J. Hilborn, O. P. Varghese, Multifunctional Hyaluronic Acid and Chondroitin Sulfate Nanoparticles: Impact of Glycosaminoglycan Presentation on Receptor Mediated Cellular Uptake and Immune Activation. ACS Appl. Mater. Inter. 8, 20614-20624 (2016).

24. J. S. Suk, Q. G. Xu, N. Kim, J. Hanes, L. M. Ensign, PEGylation as a strategy for improving nanoparticle-based drug and gene delivery. Adv. Drug Deliver. Rev. 99, 28-51 (2016).

25. E. Frohlich, The role of surface charge in cellular uptake and cytotoxicity of medical nanoparticles. Int. J. Nanomed. 7, 5577-5591 (2012). 
26. E. A. Berg, J. B. Fishman, Labeling Antibodies Using N-Hydroxysuccinimide (NHS)Fluorescein. Cold Spring Harb. Protoc. 3, 229-231 (2019).

27. X. Jin et al., A metastasis map of human cancer cell lines. Nature 588, 331-336 (2020).

28. J. A. Kim, C. Aberg, A. Salvati, K. A. Dawson, Role of cell cycle on the cellular uptake and dilution of nanoparticles in a cell population. Nat. Nanotechnol. 7, 62-68 (2012).

29. C. Aberg, J. A. Kim, A. Salvati, K. A. Dawson, Reply to 'The interface of nanoparticles with proliferating mammalian cells'. Nat. Nanotechnol. 12, 600-603 (2017).

30. E. Panet et al., The interface of nanoparticles with proliferating mammalian cells. Nat. Nanotechnol. 12, 598-600 (2017).

31. P. Rees, J. W. Wills, M. R. Brown, C. M. Barnes, H. D. Summers, The origin of heterogeneous nanoparticle uptake by cells. Nat. Commun. 10 (2019).

32. J. Barretina et al., The Cancer Cell Line Encyclopedia enables predictive modelling of anticancer drug sensitivity (vol 483, pg 603, 2012). Nature 492, 290-290 (2012).

33. M. Ghandi et al., Next-generation characterization of the Cancer Cell Line Encyclopedia. Nature 569, 503-508 (2019).

34. K. Tsuchikama, Z. Q. An, Antibody-drug conjugates: recent advances in conjugation and linker chemistries. Protein Cell 9, 33-46 (2018).

35. J. Rejman, V. Oberle, I. S. Zuhorn, D. Hoekstra, Size-dependent internalization of particles via the pathways of clathrin-and caveolae-mediated endocytosis. Biochem. $J$. 377, 159-169 (2004).

36. S. Behzadi et al., Cellular uptake of nanoparticles: journey inside the cell. Chem. Soc. Rev. 46, 4218-4244 (2017).

37. Y. Shamay et al., P-selectin is a nanotherapeutic delivery target in the tumor microenvironment. Sci. Transl. Med. 8, 345 (2018).

38. G. Saravanakumar, D. G. Jo, J. H. Park, Polysaccharide-Based Nanoparticles: A Versatile Platform for Drug Delivery and Biomedical Imaging. Curr. Med. Chem. 19, 3212-3229 (2012).

39. J. Voigt, J. Christensen, V. P. Shastri, Differential uptake of nanoparticles by endothelial cells through polyelectrolytes with affinity for caveolae. Proc. Natl. Acad. Sci. U.S.A.

111, 2942-2947 (2014). 
40. D. Szklarczyk et al., STRING v11: protein-protein association networks with increased coverage, supporting functional discovery in genome-wide experimental datasets. Nucleic Acids Res. 47, D607-D613 (2019).

41. C. von Mering, M. Huynen, D. Jaeggi, S. Schmidt, P. Bork, B. Snel, STRING: a database of predicted functional associations between proteins. Nucleic Acids Res. 31, 258-261 (2003).

42. B. Snel, G. Lehmann, P. Bork, M. A. Huynen, STRING: a web-server to retrieve and display the repeatedly occurring neighbourhood of a gene. Nucleic Acids Res. 28, 34423444 (2000).

43. D. Martin, C. Brun, E. Remy, P. Mouren, D. Thieffry, B. Jacq, GOToolBox: functional analysis of gene datasets based on Gene Ontology. Genome Biol. 5, R101 (2004).

44. M. Ashburner et al., Gene Ontology: tool for the unification of biology. Nat. Genet. 25, 25-29 (2000).

45. S. Carbon et al., The Gene Ontology resource: enriching a GOld mine. Nucleic Acids Res. 49, D325-D334 (2021).

46. K. J. Hamblett et al., SLC46A3 Is Required to Transport Catabolites of Noncleavable Antibody Maytansine Conjugates from the Lysosome to the Cytoplasm. Cancer Res. 75, 5329-5340 (2015).

47. C. K. Tsui et al., CRISPR-Cas9 screens identify regulators of antibody-drug conjugate toxicity. Nat. Chem. Biol. 15, 949-958 (2019).

48. K. Kinneer et al., SLC46A3 as a Potential Predictive Biomarker for Antibody-Drug Conjugates Bearing Noncleavable Linked Maytansinoid and Pyrrolobenzodiazepine Warheads. Clin. Cancer Res. 24, 6570-6582 (2018).

49. Q. Zhao et al., Increased expression of SLC46A3 to oppose the progression of hepatocellular carcinoma and its effect on sorafenib therapy. Biomed. Pharmacother. 114, 108864 (2019).

50. G. M. Li et al., Mechanisms of Acquired Resistance to Trastuzumab Emtansine in Breast Cancer Cells. Mol. Cancer Ther. 17, 1441-1453 (2018).

51. C. H. Adelmann et al., MFSD12 mediates the import of cysteine into melanosomes and lysosomes. Nature 588, 699-704 (2020).

52. S. Stern, B. Neun, NCL Method GTA-12. NCI Hub, doi:10.17917/YPTH-N396. 
53. M. Stephens, False discovery rates: a new deal. Biostatistics 18, 275-294 (2017).

54. R Core Team, R: A language and envrionemnt for statistical computing. R Foundation for Statistical Compouting. https://www.R-project.org/.

55. H. Wickham, ggplot2: Elegant Graphics for Data Analysis. Use R, 1-212 (2009).

56. Y. Tang, M. Horikoshi, W. X. Li, ggfortify: Unified Interface to Visualize Statistical Results of Popular R Packages. R. J. 8, 474-485 (2016).

57. M. Horikoshi, Y. Tang, ggfortify: Data Visualization Tools for Statistical Analysis Results. https://CRAN.R-project.org/package=ggfortify (2016).

\section{Acknowledgments:}

This work was supported in part by SPARC funding at The Broad Institute.

This work was also supported by a grant from the Koch Institute's Marble Center for Cancer Nanomedicine.

This work was supported in part by the Koch Institute Support (core) Grant P30-CA14051 from the National Cancer Institute.

We thank the Koch Institute's Robert A. Swanson (1969) Biotechnology Center for technical support, specifically the Flow Cytometry, High Throughput Sciences, Microscopy, and Preclinical Modeling, Imaging \& Testing cores, the Hope Babette Tang (1983) Histology Facility, and the Peterson (1957) Nanotechnology Materials Core Facility.

NB was supported by a Department of Defense Congressionally Directed Medical Research Programs Peer Reviewed Cancer Research Program Horizon Award (W81XWH-19-1-0257).

JPS was supported as a National Institutes of Health grant T32 trainee (CA136432-08) and by the Helen Gurley Brown Presidential Initiative of Dana-Farber Cancer Institute.

Fellowship support for CHA was from the NIH (NRSA F31 CA228241-01).

$\mathrm{RRC}$ is a fellow of the Parker B. Francis Foundation.

We would like to thank Todd Golub and Alex Burgin for formative feedback and helpful discussion.

We also gratefully acknowledge Thomas Diefenbach and the Ragon Institute Microscopy Core for assistance with imaging cytometry. 
Figures 1A was created in part using Biorender.com

\section{Author contributions:}

Conceptualization: NB, JPS

Methodology: NB, JPS, MK

Formal Analysis: NB, JPS, MK, MGR, MR

Investigation: NB, JPS, HCS, MGR, DR

Visualization: NB, JPS

Funding acquisition: NB, JPS, ANK, PTH

Project administration: NB, JPS, MR

Validation: NB, JPS, HCS, CHA, RRC, JHC, HL

Supervision: JAR, ANK, PTH

Writing - original draft: NB, JPS

Writing - review \& editing: NB, JPS, HCS, MK, MGR, MR, CHA, RRC, JHC, HL, JAR, ANK, PTH

Competing interests: Authors declare that they have no competing interests.

Data and materials availability: All data are available in the main text or the supplementary materials.

\section{List of Supplementary Materials}

Materials and Methods

Supplementary Text

Figs. S1 to S13

Tables S1 to S2

References (51-57)

Data $\mathrm{S} 1$ to $\mathrm{S} 2$ 